\title{
More Accurate Pose Initialization with Redundant Measurements
}

\author{
Ksenia Klionovska, Heike Benninghoff and Felix Huber \\ German Aerospace Center, Muenchener street, 20, Wessling, Germany
}

Keywords: $\quad$ Photonic Mixer Device (PMD) Sensor, 3D-2D Pose Estimation, Hough Line Transform.

\begin{abstract}
The problem described in this paper concerns the problem of initial pose estimation of a non-cooperative target for space applications. We propose to use a Photonic Mixer Device (PMD) sensor in a close range for the visual navigation in order to estimate position and attitude of the space object. The advantage of the ranging PMD sensor is that it provides two different sources of data: depth and amplitude information of the imaging scene. In this work we make use of it and propose a follow-up initial pose improvement technique with the amplitude images from PMD sensor. It means that we primary calculate the pose of the target with the depth image and then correct the pose to get more accurate result. The algorithm is tested for the set of images in the range 8 to 4.9 meters. The obtained results have shown the evident improvement of the initial pose after correction with the proposed technique.
\end{abstract}

\section{INTRODUCTION}

Computer vision is a huge branch, which allows computers to understand and process images for different applications, e.g. autonomous driving, health care, agriculture, industrial. Our research is aimed to use computer vision for the aerospace applications. The goal is to estimate an initial pose (position and attitude) of the unknown space object without any previous knowledge about it.

The state-of-the-art techniques for pose initialization in space are presented in follow works. In the article of (Sharma et al., 2018) authors propose the model based initial pose estimation of the non-cooperative spacecraft with monocular vision. The other approach (Rems et al., 2015) suggests to use LIDAR's 3D point clouds for acquisition of the unknown pose of the space object. In the follow paper (Klionovska and Benninghoff, 2017) authors show an approach for pose acquisition with the 3D data obtained from a time-of-flight Photonic Mixer Device (PMD) sensor, which can be considered as possible candidate for visual navigation in future space missions. PMD sensor provides the raster depth image of the imaging scene, which is calculated using the phase shift delay between emitted and reflected signals.

Since we continue investigating further possibility of PMD sensor, especially its robustness to estimate a pose of the space object, in this work we introduce a follow-up improvement technique for initial pose re- finement. It should be mentioned that the depth PMD sensor provides not only depth image, but also has an ability to generate co-registered amplitude data. It means: at the beginning we calculate the initial pose of the non-cooperative vehicle with the depth image, namely, with the correspondent point cloud, and after that, the obtained pose is corrected with a correspondent method using the amplitude image. The algorithm which is proposed to apply for the amplitude image consists of the image processing technique in order to detect the straight lines and end points of the lines, and successive Gauss-Newton minimization for the pose determination.

For verification of the algorithms with the PMD sensor at German Aerospace Center we run simulations with the high accuracy hardware-in-the-loop European Proximity Operations Simulator (EPOS 2.0) (Benninghoff et al., 2017) and with a prototype DLRArgos3D - P320 camera provided by Bluetechnix company. We do tests and evaluate the results for the initial pose estimation in the close range 8 to 4.9 meters.

\section{PROBLEM STATEMENT}

The problem of the initial pose estimation of the noncooperative target can be described as follows. We assume to have on board a 3D model of the target, see Figure 1, presented in a body frame. The optical pro- 
perties of the mockup's surface materials are similar with the real ones used in space. In the future we are planning to test the proposed techniques with PMD sensor using different 3D targets.
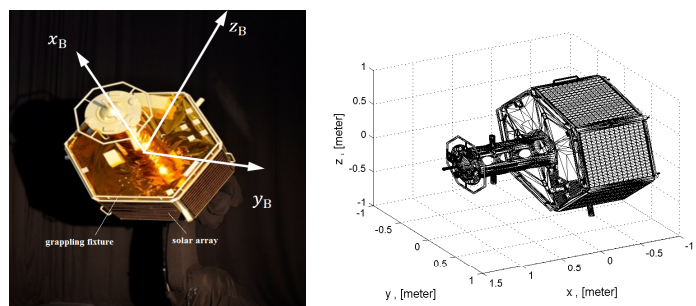

Figure 1: Image of Target in EPOS Laboratory and Its 3D Model.

The PMD sensor attached to the chaser provides co-registered depth and amplitude images of the target. The example of these images is depicted in Figure 2 . We would like to note that amplitude image can be treated as a 2D gray-scaled image. Therefore,

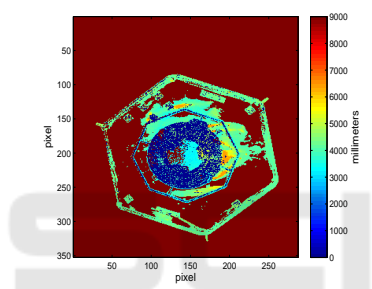

Figure 2: Depth and Amplitude Images taken with DLRArgos 3D-P320 Camera .

the task is to determine accurately the relative position vector $t_{b}$ and relative attitude of the target using only these two sources of information: 3D known model and obtained PMD image. The relative attitude defines the rotation matrix $R_{b c}$ from body coordinate frame to the camera frame. The sketch of the coordinate systems, as well as unknown position and orientation are presented in Figure 3.

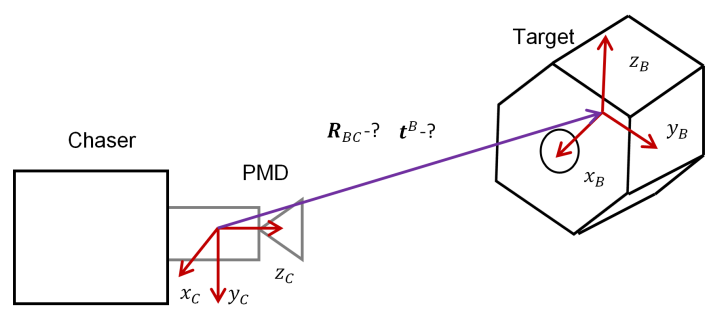

Figure 3: Coordinate Systems of the Attached PMD Sensor and Target.

\section{METHODS}

In the previous section we have determined the problem of the initial pose estimation. Since we are testing the PMD sensor for this purpose, we are going to present the sensor and its features. Further the description of the follow-up pose correction technique using an amplitude image of PMD sensor is provided.

\subsection{DLR-Argos 3D-P320 Camera}

The depth measurement principle of the PMD technologies is based on the calculation of the phase shift between the emitted NIR signal by the LED's of the camera and reflected signal from the target. The amplitude data shows the strength of the reflected signal from the object. The characteristics of the PMD sensor used in this paper are presented in Table 1 and the image of the camera is plotted in Figure 4.

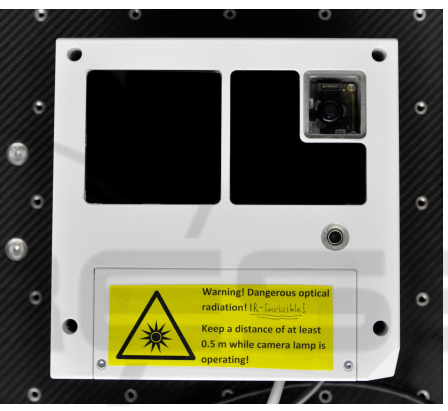

Figure 4: DLR-Argos 3D-P320 Camera in EPOS Laboratory.

Table 1: Technical Characteristics of the PMD Sensor Inside the DLR-Argos 3D-P320 Camera.

\begin{tabular}{|c|c|}
\hline Field of View (FOV) & $28.91 \times 23.45 \mathrm{deg}$ \\
\hline Resolution of the chip & $352 \times 287$ pixels \\
\hline Integration time & $24 \mathrm{~ms}$ \\
\hline Frames per second & 45 \\
\hline Modulated frequencies & $5.004 \mathrm{MHz}, 7.5 \mathrm{MHz}$, \\
& $10.007 \mathrm{MHz}, 15 \mathrm{MHz}$, \\
& $20.013 \mathrm{MHz}$, \\
& $25.016 \mathrm{MHz}, 30 \mathrm{MHz}$ \\
\hline Mass/Power consumption & $2 \mathrm{~kg} /<25.5 \mathrm{~W}$ \\
\hline
\end{tabular}

We should underline that using proposed mockup with its surfaces we are able to initialize pose only from the front side. This is because of the inability of the PMD camera to provide correct depth measurements when one is working with high absorbing surfaces, e.g solar arrays. In Figure 1 (left image), we denote solar arrays of the used mockup in the EPOS laboratory. 


\subsection{Follow-up Refinement of Initial Pose with Amplitude Image}

In the paper of Klionovska et al. (Klionovska and Benninghoff, 2017), we propose the algorithm which we used in order to acquire the pose of the noncooperative target using the depth image of PMD sensor and known 3D model. In that paper, we have discovered differednet things: the use of a proper shape (a frontal hexagon and a "nose") of the 3D model is a prerequisite for the correct work of the algorithm; the determination of the attitude of the target using only point cloud from the depth image is a demanding problem. Specifically, the determination of the target's rotation around its principal axis of inertia (in Figure 1 (left) is an axis $x_{B}$ ) only with the 3D point cloud depth data can lead to misalignments up to 30 degrees. The other rotational components can also be affected. Since it is preferable to have an accurate initial guess for the tracker in order to navigate to the target in a frame-to-frame mode, we propose an initial pose refinement with the amplitude image.

In the work of Klionovska et al. (Klionovska et al., 2018) we presented for the first time a navigation system which uses depth and amplitude images from the PMD sensor. We have shown that the use of amplitude image along with the depth image for the pose estimation leads to stable tracking, since the amplitude information can be considered as a redundant and let us calculate a pose when the depth algorithm fails or gives wrong measurements. Moreover, it was shown that (partly) lost distance information of the target from the depth images is still present in the amplitude images. It means that with the amplitude image we can get a more complete representation of the imaging target, consequently, more accurate estimation of the pose. And finally, the model-base pose estimation technique with the 2D amplitude image demonstrates more accurate estimation of the attitude of the target in comparison with the 3D pose estimation technique.

Having analyzed the pros of using the amplitude image for the pose estimation during the tracking, we have decided to apply it as a supplement processing for the enhancement of the initial pose. We assume to have the essential estimated pose of the target after pose initialization technique with the depth image. It means that the proposed technique with the amplitude image has already kind of a guess pose as an input, which is a necessary prerequisite for the chosen improvement technique. For the initial pose refinement, we are going to apply an image processing technique based on the line detection procedure with Hough Line Transform. The detected straight lines, namely the end points of that lines, will be the feature points in order to get the pose by solving 3D-2D problem. Throughout variety of the solvers (Sharma and D'Amico, 2016), here we propose to take a GaussNewton solver based on a least square minimization problem (Nocedal and Wright, 2006) (Cropp, 2001) in order to estimate the pose of the target related to the camera frame. The Gauss-Newton solver iteratively solves perspective projection equations with the known first guess. Let us consider the image processing technique and Gauss-Newton solver.

\subsubsection{Image Processing}

Since we are able to estimate the initial pose only from the front side of the mockup, the visible front hexagon is defined as an appropriate feature. The hexagon is constructed with six straight lines, which are completely observable if the target is in the FOV of the camera. The image processing pipeline in order to detect straight lines has follow steps (HoughLineTransform, 2009):

- Use of low-pass filtering to reduce image noise

- Execution of Canny-edge operator (Canny, 1986) for the edge extraction in the amplitude images

- Employment of Probabalistic Hough Line Transform for finite lines detection

The straight lines give us also the end points, which are assumed to be the detected features. Knowing the initial pose defined by the depth image, called guess pose $T_{\text {guess }}$, and calibration matrix $A$ of the PMD sensor, the 3D model can be re-projected onto the image plane, see Figure 5 (left). The calibration matrix $\mathrm{A}$ is presented by

$$
A=\left[\begin{array}{ccc}
\alpha & \gamma & u_{0} \\
0 & \beta & v_{0} \\
0 & 0 & 1
\end{array}\right]
$$

and includes the following parameters: focal lengths $\alpha$ and $\beta$, coordinates of the principal point $\left(u_{0}, v_{0}\right)$ and a skew factor $\gamma$ between $x$ and $y$ axis. We determined the calibration matrix of the current PMD sensor in the paper of Klionovska et al. (Klionovska et al., 2017) using DLR CalDe and DLR CalLab Calibration Toolbox. After 3D model re-projection, there are two sets of points in the image: detected feature points from the image and re-projected points of the 3D model. By finding nearest neighbors between them a list of feature correspondences, as in Figure 5 (right), can be generated.

\subsubsection{Gauss-Newton Solver}

The following step is to calculate the pose of the spacecraft with respect to the known 3D-2D feature cor- 

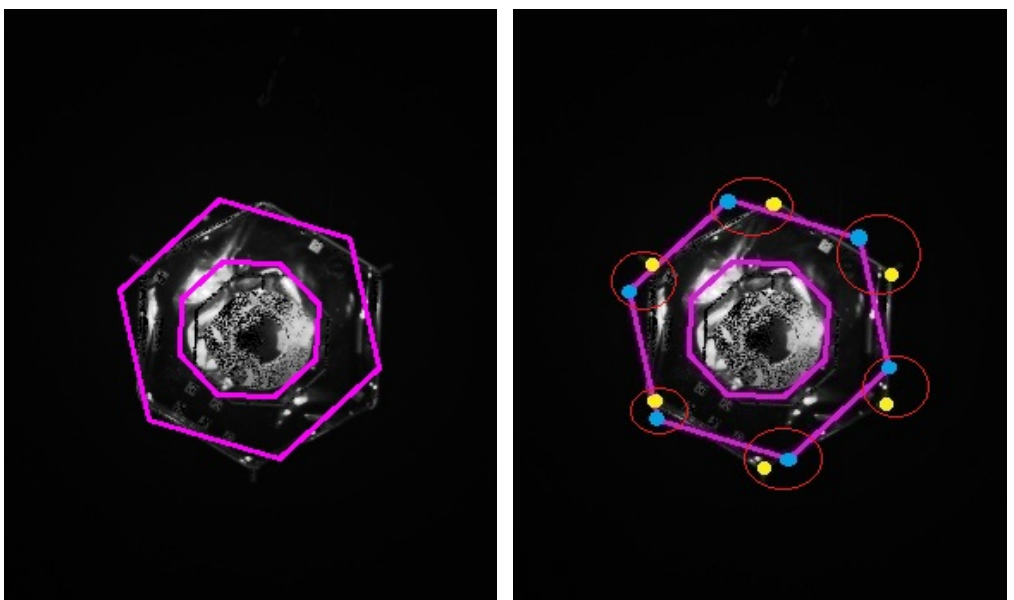

Figure 5: Left: Re-projection of the 3D Model with Pose Estimated by Depth Image. Right: Neighbors Found for Re-projected 3D Model and Detected End Points of the Image.

respondences. We assume that during image processing with the Hough Line Technique we obtained a set of image points $r_{\text {img }}=[u, v]^{T}$ and a set of corresponding model points $p^{T}=\left[p_{x}{ }^{T}, p_{y}{ }^{T}, p_{z}{ }^{T}\right]^{T}$.

Let us consider the pose $\left(R_{T}^{C}, t^{C}\right)$ as a 6 parameters vector $x=\left[t^{C^{T}}, \theta\right]$, where $t^{C^{T}}$ is position vector with respect to the camera frame, and $\theta=\left[\theta_{1}, \theta_{2}, \theta_{3}\right]$ is set of the Euler angles, which determines the orientation of the target spacecraft. The projection of the point $p^{T}$ on the image is obtained through the $3 \mathrm{D}-2 \mathrm{D}$ true perspective projection equations:

$$
\begin{gathered}
p^{C}=R_{T}^{C} p^{T}+t^{C} \\
\rho_{M}=\left[\begin{array}{l}
u \\
v
\end{array}\right]=\left[\begin{array}{l}
\frac{p_{x}{ }^{C}}{p_{z}{ }^{C}} \alpha+u_{0} \\
\frac{p_{y}{ }^{C}}{p_{z}{ }^{C}} \beta+v_{0}
\end{array}\right]
\end{gathered}
$$

In Equation 2 and Equation $3 p^{T}$ is the feature point of the target model defined in the target frame, $p^{C}$ is the same point in a camera frame after applying transformation $\left(R_{T}^{C}, t^{C}\right),(u, v)$ is the pixel of the image corresponding to the feature, $(\alpha, \beta)$ focal lengths of the camera and $\left(u_{0}, v_{0}\right)$ principal point of the camera. Equation 3 uses simple camera model, where only focal lengths and principal point of the camera are taken from Equation 1. For each coupled feature correspondence image-model it is possible to define the following residual error:

$$
e=\rho_{M}-\rho_{i m g}=\left[\begin{array}{l}
u_{M}(x)-u_{i m g} \\
v_{M}(x)-v_{i m g}
\end{array}\right]
$$

where $\rho_{M}$ is the projection of the geometric feature of the target model, whereas $\rho_{i m g}$ is the end point detected with the Hough Line Transform. The error in the Equation 4 has six unknown parameters, which are described by the state vector $x$. The state vector contains three Euler angle, which define the rotation matrix and three coordinates of the translation vector. Each feature correspondence is defined by two conditions, therefore, at least three pairs of matches between detected end points and projected features are required to solve the system equation for the defined error function. Let us assume that we have $N$ feature correspondences between image and model points. The Gauss-Newton approach iteratively minimizes the sum of square errors in order to find the position and orientation defined by $x$.

$$
S(x)=\sum_{i=1}^{N}\left\|e_{i}(x)\right\|^{2}=\sum_{i=1}^{N}\left[\left(u_{i}(x)-u_{i}\right)^{2}+\left(v_{i}(x)-v_{i}\right)^{2}\right] .
$$

Given the first guess $x_{0}$, the pose that minimizes Equation 5 is iteratively obtained as

$$
x_{k+1}=x_{k}-\left(J_{k}^{T} J_{k}\right)^{-1} J_{k}^{T} E_{k}
$$

where

$$
E_{k}=\left[\begin{array}{c}
e_{1}\left(x_{k}\right) \\
e_{2}\left(x_{k}\right) \\
\ddot{z} \\
e_{N}\left(x_{k}\right)
\end{array}\right]
$$

is the error vector with $e_{i}$ defined in the Equation 4 and $J_{k}$ is the Jacobian of $e$ calculated at $x_{k}$ and defined as

$$
J=\frac{\partial e}{\partial x} .
$$

The equation for the Jacobian (8) for point correspondences can be written as follow:

$$
J=\left[\frac{\partial e}{\partial t^{C}}, \frac{\partial e}{\partial \theta}\right]=\left[\begin{array}{ll}
\frac{\partial e_{1}}{\partial t^{C}} & \frac{\partial e_{1}}{\partial \theta} \\
\ldots . & \\
\frac{\partial e_{N}}{\partial t^{C}} & \frac{\partial e_{N}}{\partial \theta}
\end{array}\right] .
$$


The size of the Jacobian is $2 \mathrm{Nx} 6$ since each residual error in the Equation 4 is defined by two components along $u$ and $v$ coordinates of the image.

The general expression of the rows of the Jacobian being

$$
J_{i}=\left[\frac{\partial e_{i}}{\partial t^{C}}, \frac{\partial e_{i}}{\partial \theta}\right]
$$

In the Equation $10, e_{i}=\rho_{M}-\rho_{i m g}, i=1 \ldots N$.

The first element of the row can be rewritten as

$$
\frac{\partial e_{i}}{\partial t^{C}}=\frac{\partial \rho_{M}}{\partial p^{C}} \frac{\partial p^{C}}{\partial t^{C}}
$$

where

$$
\frac{\partial p^{C}}{\partial t^{C}}=I_{3 \times 3}
$$

and the follow equation obtained from Equation 2 and Equation 3

$$
\frac{\partial \rho_{M}}{\partial p^{C}}=\left[\begin{array}{ccc}
\frac{\alpha}{p_{z}^{C}} & 0 & -\frac{p_{x}^{C}}{p_{z}^{C 2}} \alpha \\
0 & \frac{\beta}{p_{z}^{C}} & -\frac{p_{y}^{C}}{p_{z}^{C 2}} \beta
\end{array}\right]
$$

Alternatively, the second element of the Equation 10 can be presented as

$$
\frac{\partial e_{i}}{\partial \theta}=\frac{\partial r_{M}}{\partial p^{C}} \frac{\partial p^{C}}{\partial \theta}
$$

with

$$
\frac{\partial p^{C}}{\partial \theta}=\left[\frac{\partial p^{C}}{\partial \theta_{1}}, \frac{\partial p^{C}}{\partial \theta_{2}}, \frac{\partial p^{C}}{\partial \theta_{3}}\right]
$$

and

$$
\frac{\partial p^{C}}{\partial \theta_{j}}=\frac{\partial R_{T}^{C}}{\partial \theta_{j}} p^{T} \quad j=1,2,3 .
$$

In the Equation 16, the rotation matrix defined in terms of Euler angles $\left[\theta_{1}, \theta_{2}, \theta_{3}\right]$ as

$$
R_{t}^{C}=\left[\begin{array}{ccc}
c \theta_{1} c \theta_{1} & s \theta_{1} s \theta_{1} & -s \theta_{2} \\
c \theta_{1} s \theta_{2} s \theta_{3}-s \theta_{1} c \theta_{3} & s \theta_{1} s \theta_{2} s \theta_{3}+c \theta_{1} c \theta_{3} & c \theta_{2} s \theta_{3} \\
c \theta_{1} s \theta_{2} s \theta_{3}+s \theta_{1} s \theta_{3} & s \theta_{1} s \theta_{2} s \theta_{3}-c \theta_{1} s \theta_{3} & c \theta_{2} c \theta_{3}
\end{array}\right]
$$

where $c \theta=\cos \theta$ and $s \theta=\sin \theta$.

\section{RESULTS}

The DLR-Argos3D - P320 sensor is able to work properly with the given mockup and chosen scenario in the range from 8 to $4.9 \mathrm{~m}$. The maximum working range 8 meters is chosen because of the camera's LED power and resolution of the sensor chip. The minimum working distance is defined in dependence on the camera FOV. If the distance is less than $4.9 \mathrm{~m}$, the whole contour of hexagon cannot be observed in some parts. We are going to test proposed follow-up refinement of the initial pose for two data sets. The first data set contains 163 images in the distance range 8 to $7 \mathrm{~m}$ from the target. The second data set contains 30 images in the distance range 7 to $4.9 \mathrm{~m}$. The bigger amount of images for the first data set is taken purposely, because we are interested to test initial pose estimation with the given PMD sensor in the far range region. As soon as we estimate the initial pose, we can start to approach the target in a frame-to-frame mode. The tracking is out of scope in this paper.

Some remarks to the evaluation of the initial pose. According to the target symmetry along its principal axis, the errors in the roll angle always lie in the range from 0 to $30 \mathrm{deg}$. Moreover, even if the errors in a roll angle are small, it can happen that the re-projection of the model's octagon onto the image doesn't match it. It means that there is a need to use any additional technique, which takes into account octagon shape for its correct determination. The numerical errors in the following sections are obtained by comparing the algorithms output and ground truth from the EPOS.

\subsection{Distance Range 8 to 7 Meters}

We execute the pose initialization technique with the depth images and thereafter run the follow-up initial pose refinement with the amplitude images for the first data set. In Table 2 the mean errors in the calculated initial pose with and without correction are presented. The ground truth from the EPOS facility was use for calculation of the errors.

Table 2: Mean Error of the Initial Estimated Pose with and without Correction for the First Data Set.

\begin{tabular}{|c|c|c|}
\hline mean value & without correction & with correction \\
\hline$\mu_{\text {roll }}$, deg & 13.387 & 0.788 \\
\hline$\mu_{\text {pitch }}$, deg & 1,960 & 0.492 \\
\hline$\mu_{\text {yaw }}$, deg & 1,594 & 0.525 \\
\hline$\mu_{z}, m$ & 0.1293 & 0.0805 \\
\hline$\mu_{y}, m$ & 0.0436 & 0.0201 \\
\hline$\mu_{x}, m$ & 0.0226 & 0.0108 \\
\hline
\end{tabular}

From the Table 2 one can observe more accurate estimation of the attitude and position of the initial pose after application of the initial pose refinement with amplitude image. In Figure 6 (down) it can be noticed that in many cases we cannot properly define the initial rotation of the target around it's principle axis using only depth information. Nevertheless, this never happens after initial pose refinement, see Figure 7 (down). The mean value for the roll angle $\mu_{\text {roll }}$ of the essential initial pose reaches $13.387 \mathrm{deg}$ and the maximum $29.87 \mathrm{deg}$, whereas the $\mu_{\text {roll }}$ value for the corrected pose is $0.788 \mathrm{deg}$ and the maximum value 
reached $3.207 \mathrm{deg}$. Concerning the estimation of the initial position in the range from 8 to $7 \mathrm{~m}$, one can observe that the mean error $\mu_{z}$ for the distance measurements between target and chaser without correction is $0.1293 \mathrm{~m}$, and for the corrected pose it is 0.0805 meters. For the position errors along two other axis $y$ and $x$ the mean errors $\mu_{y}$ and $\mu_{x}$ are two times less than for the corrected initial pose, see Table 2. In Figure A 2 we present some amplitude images in the range from 8 to $7 \mathrm{~m}$ with the resulted poses of the target. Every row contains two pairs of poses: calculated initial pose without correction and refined pose with the amplitude.
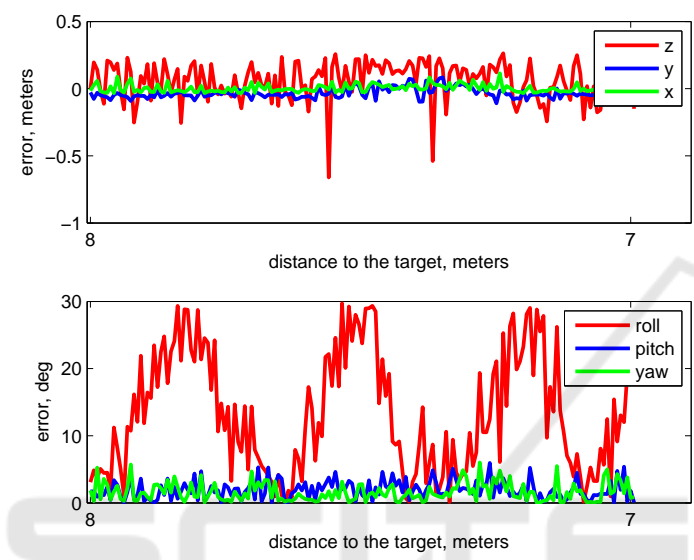

Figure 6: Translation and Rotation errors for the initial estimated pose only with the depth data in the range from 8 to 7 meters.
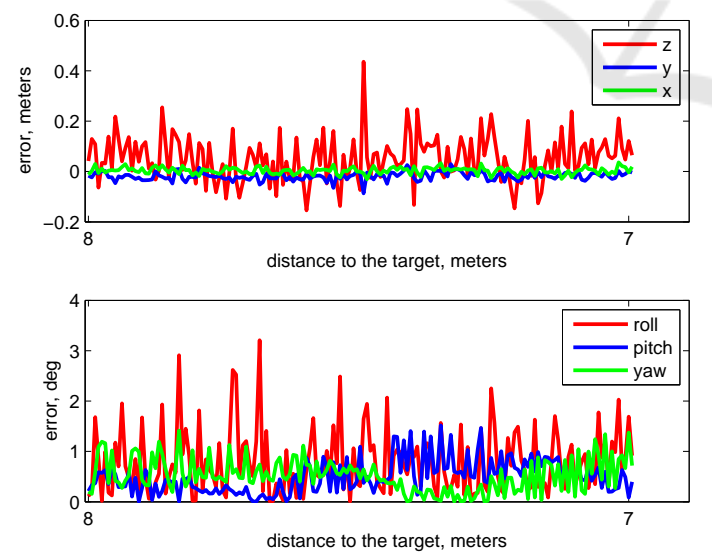

Figure 7: Translation and Rotation errors for the corrected initial estimated pose in the range from 8 to 7 meters.

\subsection{Distance Range 7 to 4.9 Meters}

Let us consider the results of the initial pose estimation of the second data set with and without correections in the range from 7 to $4.9 \mathrm{~m}$. It should be noticed that the closer the camera to the target, the bigger the size of the point cloud. In our previous paper (Klionovska and Benninghoff, 2017), where we discussed the pose initial algorithm with the depth data, it was mentioned the fact that the depth algorithm is sensitive to the size of the point cloud. If the point cloud of a scene has a big amount of points, it can happen that the accuracy of the initial pose drops. This is due to the fact that the close to the target, more details can be observed and measured by the camera. The scene point cloud will be more dense than the model, and this could lead to some misalignment. Actually, we can see this in Figure 8 (down), where the errors in determining yaw and pitch angles for the second data set are evidently higher than in the first data set in Figure 6 (down). From the Table 3, where the mean errors are summed up for the position and orientation of the estimated initial poses, one can notice the significant advantages of the follow-up initial pose correction.

Table 3: Mean Error of the Initial Estimated Pose with and without Correction for the Second Data Set.

\begin{tabular}{|c|c|c|}
\hline mean value & without correction & with correction \\
\hline$\mu_{\text {roll }}$, deg & 11.304 & 0.821 \\
\hline$\mu_{\text {pitch }}$, deg & 3.989 & 0.682 \\
\hline$\mu_{\text {yaw }}$, deg & 5.450 & 0.760 \\
\hline$\mu_{z}, m$ & 0.1581 & 0.0972 \\
\hline$\mu_{y}, m$ & 0.0157 & 0.0157 \\
\hline$\mu_{x}, m$ & 0.0224 & 0.0109 \\
\hline
\end{tabular}

Let us consider one case in more details. In Figure A2, we again print some pairs of amplitude images and the resulted pose calculated with and without correction for the second data set. Two images from the left of the last row in Figure A2 reflect initial pose at the distance $4.9 \mathrm{~m}$. The attitude errors of the uncorrected initial pose occur for the roll, pitch and yaw angles are $23.562 \mathrm{deg}, 0.5333 \mathrm{deg}$ and $4.734 \mathrm{deg}$. The position errors along $x, y$ and $z$ axis are 0.0231 $m, 0.0034 \mathrm{~m}, 0.0006 \mathrm{~m}$. The errors for the corrected pose: roll - $0.669 \mathrm{deg}$, pitch - $1.160 \mathrm{deg}$ and yaw $0.471 \mathrm{deg}$; position along $x, y$ and $z$ axis are 0.0009 $m, 0.0236 m, 0.1016 m$. Having a look at Figure 8 and Figure 9 one can notice that this was exactly an unique case, when the depth coordinate without correction was calculated extremely accurate in comparison with the corrected result. Nevertheless, the mean value $\mu_{z}=0.0109 \mathrm{~m}$ for the corrected initial pose is much better than the $\mu_{z}=0.0224 \mathrm{~m}$ for the primary estimated pose.

\section{CONCLUSION}

In this paper, we presented the improvement technique for the initial pose estimation of the non- 

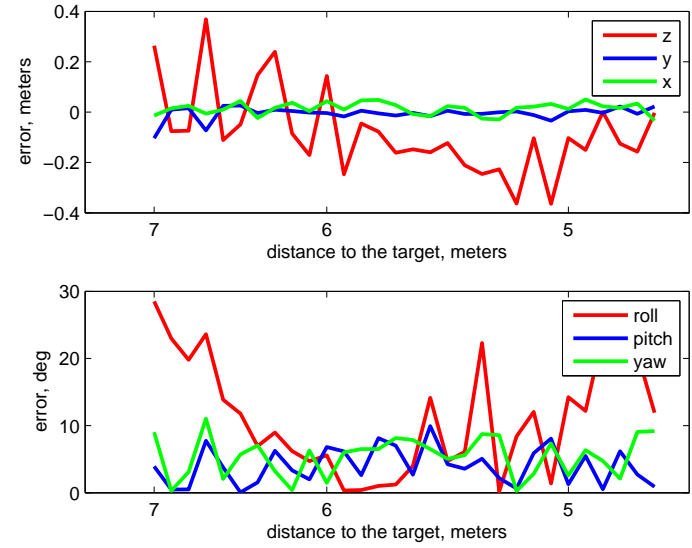

Figure 8: Translation and Attitude Errors for the Initial Estimated Pose only with Depth Data in the Range from 7 to 4.9 Meters.
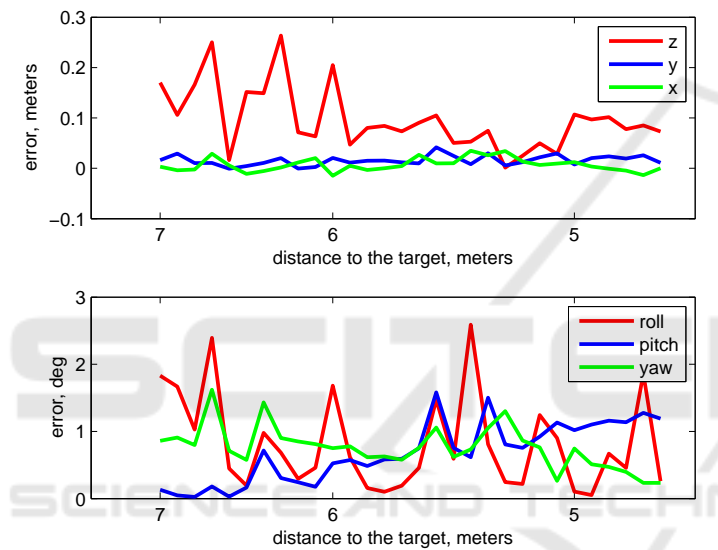

Figure 9: Translation and Attitude Errors for the Corrected Initial Estimated Pose in the Range from 7 to 4.9 Meters.

cooperative target for space missions with relatively new time-of-flight PMD sensor. The proposed approach takes into account the additional amplitude data of the PMD sensor provided in parallel to the depth measurements. This feature leads to the software redundancy without hardware redundancy. As soon as a primary initial pose can be calculated with the depth image, the following pose refinement technique takes place for more accurate acquisition of the position and orientation of the unknown target using a single PMD sensor. Conducting experiments with the real images of PMD sensor and the existent mockup, we have shown the necessity of the follow-up initial pose refinement, since it crucially increases the accuracy of the estimated pose.

\section{REFERENCES}

Benninghoff, H., Rems, F., and Risse, E. (2017). European proximity operations simulator 2.0 (EPOS) a robotic-based rendezvous and docking simulator. Journal of large-scale research facilities, 3 .

Canny, J. (1986). A computational approach to edge detection. IEEE Transactions on Pattern Analysis and Machine Intelligence, (6).

Cropp, A. (2001). Pose estimation and relative orbit determination of a nearby target microsatellite using passive imagery. PhD Thesis, University of Surrey.

HoughLineTransform (2009). Hough Line Transform. Available at http: //web.ipac.caltech.edu/staff/fmasci/home/ /astro_refs/HoughTrans_lines_09.pdf .

Klionovska, K. and Benninghoff, H. (2017). Initial pose estimation using PMD sensor during the rendezvous phase in on-orbit servicing missions. 27th AAS/AIAA Space Flight Mechanics Meeting, Texas, USA.

Klionovska, K., Benninghoff, H., and Strobl, K. H. (2017). PMD Camera-and Hand-Eye-Calibration for On-Orbit Servicing Test Scenarios On the Ground. 14th Symposium on Advanced Space Technologies in Robotis and Automation (ASTRA),Leiden, the Netherlands.

Klionovska, K., Ventura, J., Benninghoff, H., and Huber, F. (2018). Close range tracking of an uncooperative target in a sequence of photonic mixer device (pmd) images. Robotics, 7.

Nocedal, J. and Wright, S. (2006). Numerical optimization. Springer-Verlag New York.

Rems, F., Moreno Gonzalez, J. A., Boge, T., Tuttas, S., and Stilla, U. (2015). Fats initial pose estimation of spacecraft from lidar point cloud data. 13th Symposium on Advanced Space Technologies in Robotics and Automation.

Sharma, S. and D'Amico, S. (2016). Comparative assessment of techniques for initial pose estimation using monocular vision. Acta Astronautica, 123.

Sharma, S., Ventura, J., and D'Amico, S. (2018). Robust model-based monocular pose initialization for noncooperative spacecraft rendezvous. Journal of Spacecraft and Rockets, pages 1-16.

\section{APPENDIX}

Assumptions: Column I is a initial pose without correction. Column II is a initial pose corrected with the proposed technique. 

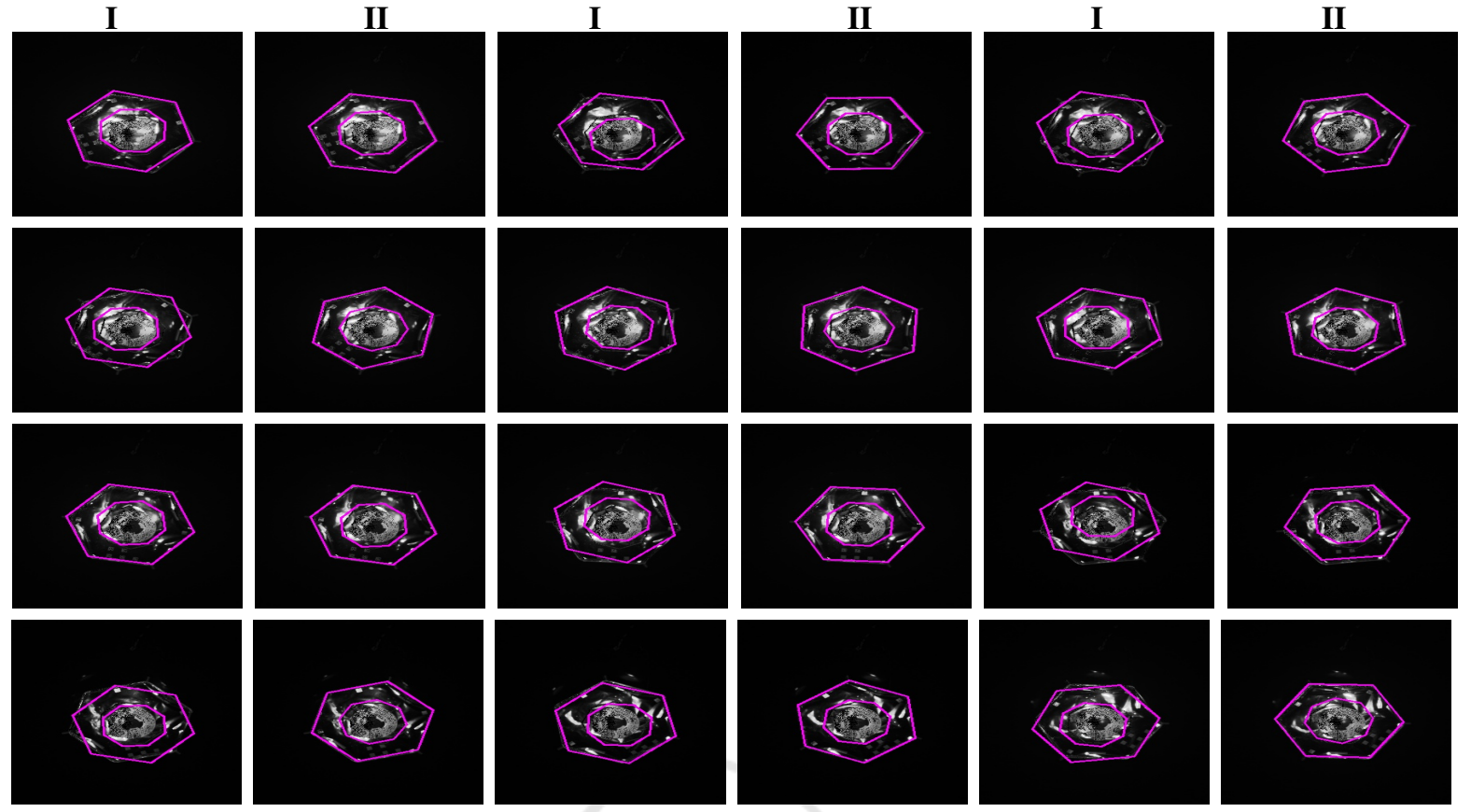

Figure A1: Set of Some Amplitude Images in Range 8 to 7 Meters with Uncorrected and Corrected Initial Poses.
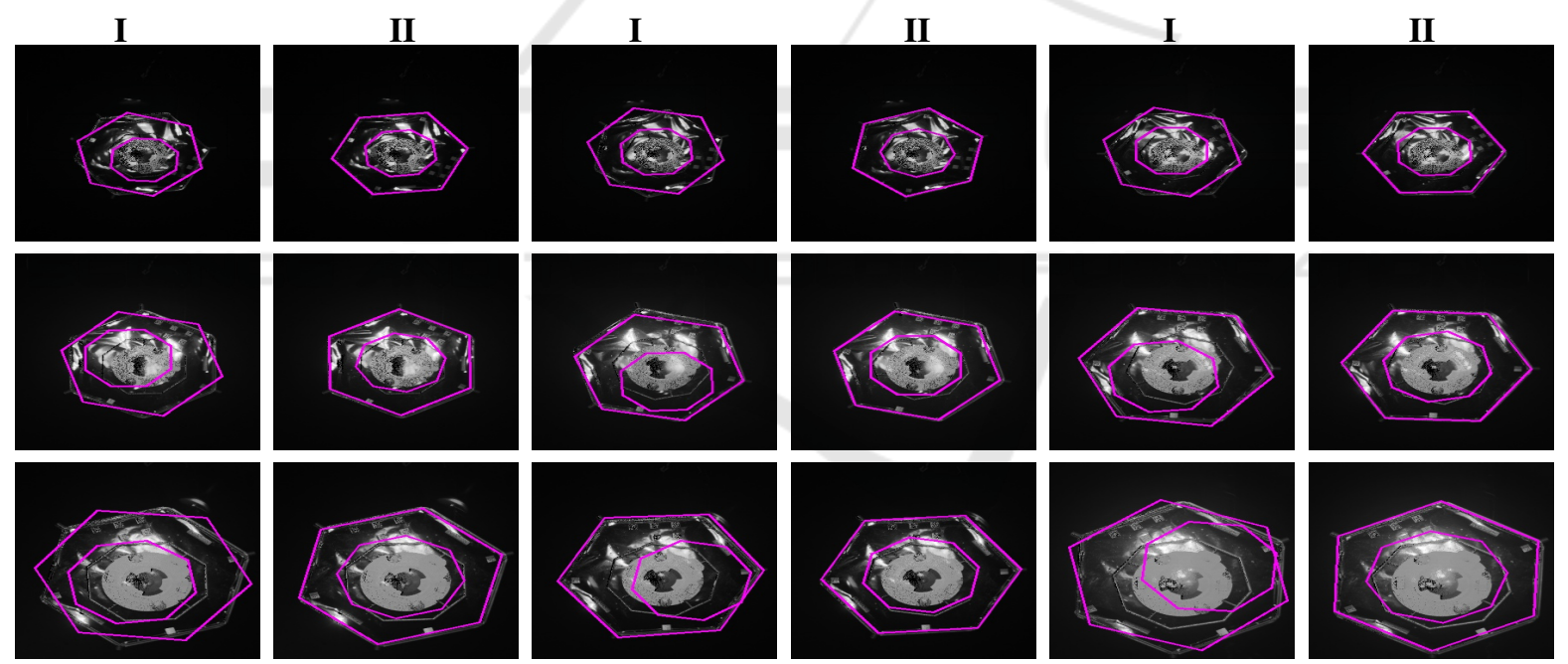

Figure A2: Set of Some Amplitude Images in Range 7 to 4.9 Meters with Uncorrected and Corrected Initial Poses. 